\title{
Educação em saúde nos livros didáticos de Ciências e Biologia brasileiros: um panorama das teses e dissertações (1994 - 2018)
}

\author{
Karine Rudek \\ Mestre em Ensino de Ciências (PPGEC), Universidade Federal da Fronteira Sul (UFFS), \\ campus Cerro Largo \\ $\triangle$ rudekkarine@gmail.com \\ Erica do Espirito Santo Hermel \\ Professora do Quadro Permanente do Programa de Pós-Graduação em Ensino de Ciências (PPGEC), \\ Universidade Federal da Fronteira Sul (UFFS), campus Cerro Largo \\ Doutora em Ciências Biológicas: Neurociências/UFRGS
}

\begin{abstract}
Resumo:
O artigo apresenta um panorama da produção acadêmica de como a Educação em Saúde tem sido apresentada nos livros didáticos (LDs) do Ensino Fundamental e Médio em teses e dissertações desenvolvidas no período entre 1994 e 2018. Foram selecionadas e investigadas 9 pesquisas que abordam a temática Educação e abordagens de Saúde nos LDs de Ciências e Biologia. Como metodologia de pesquisa usamos Análise de Conteúdo. Os resultados obtidos mostram que a maioria das produções são dissertações (7). Quanto as abordagens de saúde, a biomédica se fez presente em todas as produções (9), seguida da comportamental (5) e da socioecológica (3). Evidenciamos que a Educação em Saúde está fortemente ligada ao Ensino de Ciências e Biologia e que os conceitos de saúde abordados nos LDs contribuem de forma muito frágil com abordagens mais críticas de saúde. O estudo apresenta a preocupação em discutir a saúde desde o início da escolarização. A pesquisa apontou também para a carência de discussões em Programas de Pós-graduação sobre a temática Educação em Saúde e a necessidade da inserção da temática nos LDs de forma mais crítica e reflexiva, ressaltando a necessidade da divulgação e disseminação desta temática visando abrir novos campos para discussão e reflexão sobre a Educação em Saúde no contexto escolar.

Palavras-chave: Abordagens da Saúde, Currículo, Ensino de Ciências e Biologia.
\end{abstract}

\section{Health education in Brazilian science and biology textbooks: an overview of theses and dissertations (1994 - 2018)}

\begin{abstract}
:
The article presents an overview of the academic production of how Health Education has been presented in elementary and high school textbooks (LDs) in theses and dissertations developed between 1994 and 2018. Nine researches were selected and investigated that address the theme Health education and approaches in the Science and Biology LDs. As a research methodology we use Content Analysis. The results obtained show that most productions are dissertations (7). As for health approaches, biomedical was present in all productions (9), followed by behavioral (5) and socioecological (3). We show that Health Education is strongly linked to Science and Biology Education and that the health concepts covered in LDs contribute very fragile to more critical health approaches. The study is concerned with discussing health from the beginning of schooling. The research also pointed to the lack of discussions in Postgraduate Programs on the theme of Health Education and the need to insert the theme in the LDs in a more critical and reflective way, emphasizing the need to disseminate and disseminate this theme in order to open new fields for discussion and reflection on Health Education in the school context.
\end{abstract}


Keywords: Health Approaches, Curriculum, Science Teaching and Biology.
La educación para la salud en los libros de texto de ciencia y biología brasileños: una visión general de tesis y disertaciones (1994 - 2018)

\section{Resumen:}

El artículo presenta un panorama de la producción académica de cómo la Educación para la Salud se ha presentado en los libros de texto (LD) de educación básica y media en las tesis y disertaciones desarrolladas entre 1994 y 2018. Se seleccionaron e investigaron nueve investigaciones que abordan el tema Educación y enfoques en salud en los LD de Ciencias y Biología. Como metodología de investigación utilizamos el análisis de contenido. Los resultados obtenidos muestran que la mayoría de las producciones son disertaciones (7). En cuanto a los enfoques de salud, el biomédico estuvo presente en todas las producciones (9), seguido del conductual (5) y socioecológico (3). Mostramos que la Educación para la Salud está fuertemente vinculada a la Educación en Ciencias y Biología y que los conceptos de salud cubiertos en los LD contribuyen de manera muy frágil a enfoques de salud más críticos. El estudio se ocupa de discutir la salud desde el inicio de la escolarización. La investigación también señaló la falta de discusiones en los Programas de Posgrado sobre el tema de Educación para la Salud y la necesidad de insertar el tema en los LD de una manera más crítica y reflexiva, enfatizando la necesidad de la difusión y difusión de este tema con el fin de abrir nuevos campos de discusión y reflexión sobre la Educación para la Salud en el contexto escolar.

Palabras clave: Enfoques de salud, pan de estúdios, enseñanza de las ciências y biologia.

\section{INTRODUÇÃO}

No Brasil, o campo de pesquisa em Educação em Saúde vem se desenvolvendo consideravelmente nas últimas décadas. Os debates sobre Educação em Saúde começaram a ser realizados de forma obrigatória nos currículos escolares em 1971, por meio da Lei Federal no 5.692, que visava a uma abordagem interdisciplinar da saúde (BRASIL, 1971). As leis nacionais que orientam a educação sinalizam a saúde como um tema que deve ser trabalhado de forma interdisciplinar e transversal. Diante disso, pesquisas (MARTINS, 2011; MARTINS; SANTOS; EL-HANI, 2012) apontam para a predominância do modelo biomédico de saúde, que caracteriza a saúde como ausência de doença. No entanto, outras abordagens têm se destacado com o passar dos anos, como a comportamental e a socioecológica, abordada mais recentemente.

A saúde é de extrema importância para a vida. Ela permeia o cotidiano, as relações interpessoais, os discursos midiáticos, fazendo parte do processo de escolarização. Pesquisas anteriores, realizadas por Mohr (2002), Zancul e Costa (2012) e Martins (2017), discutem a importância de um olhar mais crítico ao conceito de saúde nos LDs, sendo relevante ampliar as abordagens sobre Educação em Saúde, pensando em uma discussão global do conceito. 
Os LDs aproximam da sala de aula conceitos e discussões de Educação em Saúde, na maioria das vezes podendo ser a única forma de acesso a ela. Segundo afirma Rosa (2018.p2) “[...] o LD é um material formulado com um propósito específico, que é dar suporte aos processos pedagógicos [...]", dessa forma, os professores utilizam este material para planejamento e utilizando-os em suas aulas. Ainda, Rosa (2018, p.13) dialoga sobre as escolhas feitas pelos professores sobre determinados LDs “[...] ao fazer uma escolha por determinado LD, de certa forma também realiza uma opção por determinada concepção de ensinar sua disciplina, além de determinadas visões de professor, de aluno, de escola, de Ciências e de currículo". Considerando que os LDs são elementos representativo do currículo escolar, mesmo nos dias atuais.

Muitas vezes, a maneira em que os autores abordam os conteúdos nos LDs podem induzir a equívocos no aprendizado. Segundo Delizoicov (2006), o qual dialoga sobre a organização dos conteúdos nos LDs, determinadas abordagens discutidas pelos autores podem contribuem para a construção de visões simplistas em determinados contextos, dialogando assim com abordagens tradicionais como a biomédica. Assim, torna-se importante analisar os LDs, verificando quais as abordagens de saúde estão presentes, bem como analisar e identificar as evoluções conceituais presentes nas obras.

[...] os livros são organizados com o objetivo de familiarizar rapidamente o estudante com a estrutura conceitual de um determinado campo do saber. Como consequência são realizadas simplificações e apenas os resultados de todo um processo de produção do conhecimento é apresentado (DELIZOICOV, 2006, p. 265).

Vasconcelos e Souto (2007, p. 93-94) ressaltam, em suas publicações, a importância da investigação nos LDs: “[...] no ensino de ciências, os livros didáticos constituem um recurso de fundamental importância, já que representam em muitos casos, o único material de apoio didático disponível para alunos e professores". Neste contexto investigativo, Martins e Brito (2006, p. 259) alertam para alguns problemas que podem ser considerados como um obstáculo para o ensino: “os autores apresentam narrativas simplificadas, omitindo aspectos importantes [...], o que impede a percepção de que o processo de construção do pensamento científico é complexo e inclui erros e acertos". A simplificação do conteúdo e a carência em abordagens mais significativas podem prejudicar o aluno quando utiliza o recurso didático para estudos. 
A Educação em Saúde tem se tornado importante na construção de novos conhecimentos, bem como na construção de estilos de vida saudáveis que visam à promoção da saúde. Assim, a investigação de como essa temática vem sendo desenvolvida nos LDs se faz necessária, tendo em vista a relevância desse recurso para motivar o aprendizado e auxiliar os estudantes a se tornarem competentes na adoção de práticas comportamentais visando o conhecimento adquirido em sala de aula.

Partimos da necessidade de se ter uma visão geral do que vem sendo produzido em programas de pós-graduação stricto sensu sobre a temática Educação em Saúde nos LDs, para assim compreender a evolução das pesquisas, a partir do levantamento das produções acadêmicas na Biblioteca Digital Brasileira de Teses e Dissertações (BDTD), localizada no órgão nacional do Instituto Brasileiro de Informação em Ciência e Tecnologia (IBICT).

A escolha do tipo de pesquisa foi definida por permitir uma coleta dos documentos produzidos sobre o tema, possibilitando uma visão do que outros pesquisadores já publicaram e, assim, trazendo elementos para futuras análises e reflexões sobre o desenvolvimento acadêmico da área. Assim, buscamos compreender quais as abordagens de saúde (biomédica, comportamental e socioecológica) estão sendo apresentadas nos LDs de Ciências e Biologia, realizando um levantamento dos principais trabalhos sobre o assunto publicados no período de 1994 a 2018.

\section{METODOLOGIA}

A presente pesquisa em Ensino de Ciências apresenta uma abordagem qualitativa, com análise do tipo documental (LÜDKE; ANDRÉ, 2013), realizada a partir de revisão bibliográfica em trabalhos acadêmicos brasileiros disponíveis no BDTD. O mapeamento teve o objetivo de criar uma visão do cenário dessa temática de pesquisa nos últimos 24 anos (1994 $-2018)$.

Os descritores utilizados foram: Abordagens de Educação em Saúde e LDs. Buscando atender os objetivos deste estudo, destacamos que, de um total de 137 trabalhos, entre teses e dissertações, que abordam ambas as temáticas, após uma análise sistemática, a fim de 
identificar a partir da leitura dos títulos, dos resumos e das palavras-chaves, selecionamos 9 trabalhos, sendo 2 teses e 7 dissertações, que abordam discussões sobre: "Educação em saúde nos LDs de Ciências e Biologia".

Identificamos os trabalhos acadêmicos da seguinte forma: para dissertações designamos D1, D2 ... D7 e para as teses T1 e T2. E, a fim de facilitar a compreensão dos resultados, os excertos retirados dos textos, como exemplos, foram colocados em itálico. Para a análise qualitativa do material, usamos a Análise de Conteúdo proposta por Bardin (2004), que compreende as três seguintes etapas: Pré-análise, Exploração do material e Análise dos dados, que compreende o tratamento dos resultados, a fim de identificar nas teses e dissertações as concepções e abordagens de saúde (biomédica, comportamental e socioecológica), que estão sendo apresentadas nos LDs de Ciências e Biologia (Quadro 1).

Quadro 1 - Abordagens de saúde.

\begin{tabular}{|c|l|}
\hline ABORDAGENS DE SAÚDE & \multicolumn{1}{c|}{ CONCEITO } \\
\hline Biomédica & $\begin{array}{l}\text { A saúde é discutida em oposição à doença, o tratamento e a } \\
\text { cura do corpo são privilegiados, e as influências sobre a saúde } \\
\text { oriundas de níveis mais elevados do que o biológico, como os } \\
\text { níveis social, cultural e psicológico, são negligenciadas } \\
\text { (CARVALHO et al., 2007). }\end{array}$ \\
\hline Comportamental & $\begin{array}{l}\text { Está interessada principalmente em alterar os padrões } \\
\text { individuais de exposição ao risco, por meio das chamadas } \\
\text { "mudanças comportamentais" (CASTIEL, 2004). }\end{array}$ \\
\hline Socioecológica & $\begin{array}{l}\text { A condição de adoecimento se estabelece quando o indivíduo } \\
\text { tem um desequilíbrio que afeta/relaciona os aspectos físicos, } \\
\text { mentais e socioambientais. Visão positiva e coletiva de saúde, } \\
\text { a saúde é entendida como o bem-estar biopsicossocial e } \\
\text { ambiental. (WESTPHAL, 2006) }\end{array}$ \\
\hline
\end{tabular}

Fonte: Elaborado pelas pesquisadoras

\section{RESULTADOS: APRESENTAÇÃO E DISCUSSÃo}

Após a pré-análise e exploração do material, nesta terceira etapa, buscamos apresentar os dados e interpretações a que chegamos nesta pesquisa, de forma simples, 
identificar em teses e dissertações a presença das abordagens de saúde em LDs de Ciências e Biologia (Quadro 2). Portanto, diante do objetivo de apresentar o panorama da produção acadêmica sobre Educação em Saúde nos LDs. Foram encontradas 9 produções acadêmicas que abordam a temática, das quais 2 teses e 7 dissertações, defendidas e publicadas no período de 1994 a 2018. Na região Sudeste, os estados de São Paulo e Rio de Janeiro são os que mais apresentam publicações relacionadas à temática Educação em saúde em LDs de Ciências e Biologia, com cinco trabalhos distribuídos entre as instituições de ensino FGV (1), UNESP (2), USP (1), FIOCRUZ (1). A região Sul (Rio Grande do Sul e Santa Catarina) apresenta dois trabalhos publicados, sendo um da UNIJUí e outro da UFSC. Já na região Nordeste, o único estado que apresentou discussões neste contexto foi a Bahia, com também dois trabalhos oriundos da UFBA.

Quadro 2 - Teses e Dissertações com a temática Educação em Saúde nos LDs.

\begin{tabular}{|c|c|c|}
\hline CóDIGO & TÍTULO & $\begin{array}{c}\text { ABORDAGEM DE } \\
\text { SAÚDE }\end{array}$ \\
\hline D1 & $\begin{array}{l}\text { MOHR, Adriana. A Saúde na Escola: análise de livros } \\
\text { didáticos de } 1^{\mathrm{a}} \text { a } 4^{\mathrm{a}} \text { séries. } 1994.100 \mathrm{f} \text {. Dissertação } \\
\text { (Mestrado) - Curso de Mestrado em Educação, Fundação } \\
\text { Getúlio Vargas, Rio de Janeiro, } 1994 .\end{array}$ & Biomédica (sanitária) \\
\hline D2 & $\begin{array}{l}\text { CASAGRANDE, Grasiela de Luca. A genética humana no } \\
\text { livro didático de biologia. 2006. 121 f. Dissertação } \\
\text { (Mestrado) - Curso de Programa de Pós-graduação em } \\
\text { Educação Científica e Tecnológica (PPGECT), } \\
\text { Universidade Federal de Santa Catarina, Florianópolis, } \\
2006 \text {. }\end{array}$ & Biomédica \\
\hline D3 & $\begin{array}{l}\text { TANAKA, Cláudio. Análise do conteúdo sobre Saúde } \\
\text { Bucal no material didático de Ciências de } \mathbf{1}^{\mathrm{a}} \text { a } \mathbf{4}^{\mathrm{a}} \text { série } \\
\text { de escolas de Ensino Fundamental de Araçatuba - } \\
\text { SP. 2006. } 78 \text { f. Dissertação (Mestrado) - Curso de } \\
\text { Programa de Pós- graduação em Odontologia Preventiva } \\
\text { e Social da Faculdade de Odontologia de Araçatuba, } \\
\text { Universidade Estadual Paulista Júlio de Mesquita Filho, } \\
\text { Araçatuba, 2006. }\end{array}$ & Comportamental \\
\hline D4 & $\begin{array}{l}\text { CARVALHO, Fausto Flor. Acidentes infantis: relatos de } \\
\text { diretores e professores de ensino fundamental e análise } \\
\text { do material didático. 2008.103 f. Dissertação (Mestrado) - } \\
\text { Curso de Faculdade de Filosofia e Ciências, Unesp - } \\
\text { Universidade Estadual Paulista "Júlio de Mesquita Filho", } \\
\text { Marília - Sp, 2008. }\end{array}$ & $\begin{array}{l}\text { Biomédico } \\
\text { Comportamental }\end{array}$ \\
\hline
\end{tabular}




\begin{tabular}{|c|c|c|}
\hline D5 & $\begin{array}{l}\text { CICCO, Roberta Ribeiro de. Potencialidades e limites do } \\
\text { ensino das doenças sexualmente transmissíveis: um } \\
\text { estudo qualitativo na perspectiva socioantropologicas. } \\
\text { 2012. } 206 \mathrm{f} \text {. Dissertação (Mestrado) - Curso de Mestrado } \\
\text { em Ensino em Biociências e Saúde, Instituto Oswaldo } \\
\text { Cruz, Rio de Janeiro, 2012. }\end{array}$ & Biomédica \\
\hline D6 & $\begin{array}{l}\text { BERNARD, Aline. Promoção da alimentação saudável } \\
\text { no contexto do currículo escolar. } 2016.129 \text { f. } \\
\text { Dissertação (Mestrado) - Curso de Programa de Pós- } \\
\text { graduação em Educação nas Ciências, Unijuí - } \\
\text { Universidade Regional do Noroeste do Estado do Rio } \\
\text { Grande do Sul, Ijuí, 2016. }\end{array}$ & Biomédica \\
\hline D7 & $\begin{array}{l}\text { TORRES, Camile da Silva. Abordagens de saúde em } \\
\text { livros didáticos de biologia: reflexões sobre a saúde da } \\
\text { população negra. 2018. } 144 \mathrm{f} \text {. Dissertação (Mestrado) - } \\
\text { Curso de Programa de Pós-graduação em Ensino, Filosofia } \\
\text { e História das Ciências, Universidade Federal da Bahia } \\
\text { Universidade Estadual de Feira de Santana, Salvador - } \\
\text { Bahia, 2018. }\end{array}$ & $\begin{array}{l}\text { Biomédica } \\
\text { Comportamental } \\
\text { Socioecológica }\end{array}$ \\
\hline T1 & $\begin{array}{l}\text { MONTEIRO, Paulo Henrique Nico. A saúde nos livros } \\
\text { didáticos no Brasil: concepções e tendências nos anos } \\
\text { iniciais do Ensino Fundamental. 2012. } 210 \text { f. Tese } \\
\text { (Doutorado) - Curso de Faculdade de Educação, } \\
\text { Universidade de São Paulo, São Paulo, 2012. }\end{array}$ & $\begin{array}{l}\text { Biomédica } \\
\text { Comportamental } \\
\text { Socioecológica } \\
\text { (biopsicossocial) }\end{array}$ \\
\hline $\mathrm{T} 2$ & $\begin{array}{l}\text { MARTINS, Liziane. Abordagens da saúde em livros } \\
\text { didáticos de biologia: análise crítica e proposta de } \\
\text { mudança. 2017. } 165 \text { f. Tese (Doutorado) - Curso de } \\
\text { Programa de Pós-graduação em Ensino, Filosofia e } \\
\text { História das Ciências, Universidade Federal da Bahia - } \\
\text { Ufba Universidade Estadual de Feira de Santana - Uefs, } \\
\text { Salvador - Bahia, 2017. }\end{array}$ & $\begin{array}{l}\text { Biomédica } \\
\text { Comportamental } \\
\text { Socioecológica }\end{array}$ \\
\hline
\end{tabular}

Fonte: Elaborado pelas pesquisadoras

Em D1, a autora menciona no título ambas as temáticas investigadas (Educação em saúde e LDs): “A Saúde na Escola: análise de livros didáticos de lª a $4^{\underline{a}}$ séries” (grifos nossos). A investigação parte de uma análise e discussão sobre os conceitos de saúde presentes nos LDs dos anos iniciais, sendo as três coleções mais compradas pela Fundação de Assistência ao Estudante do Ministério da Educação e do Desporto (MEC), participantes do Programa Nacional do Livro Didático (PNLD), de 1991, no estado do Rio de Janeiro. A autora cita o conceito biomédico como o mais presente, caracterizando-o como enfoque sanitarista, abordando a prevenção de doenças ou ausência das mesmas como conceito de saúde: "O enfoque sanitário privilegiado na presente coleção, excetuando-se os conteúdos relativos aos primeiros 
socorros, é exclusivamente preventivo [...] o enfoque sanitário é, como na coleção anterior, majoritariamente preventivo" (D1, p. 61).

O estudo aponta que o conceito biomédico de saúde se sobrepõe nas três coleções investigadas. A autora sinaliza, em sua análise, a constante abordagem da doença como contagiosa e a conceituação de saúde como a ausência de doença ou acidentes. Na abordagem biomédica, a saúde é entendida em oposição à doença e atividades preventivas são oriundas desta perspectiva sanitarista. De acordo com Tesser e Luz, “[...] nesse tipo de abordagem [...] o olhar sobre a saúde e a prática médica é alicerçado na doença” (2002, p. 366).

Em D2, a autora analisa como as doenças genéticas estão inseridas nos LDs de Biologia. O estudo desta temática foi investigado nos 14 LDs de Biologia mais utilizados pelos professores de Florianópolis, bem como nos livros utilizados nas referências bibliográficas para as provas de Biologia do vestibular de duas Instituições de Ensino Superior de Santa Catarina (UFSC e UDESC).

Segundo a autora, os LDs não apresentam o conteúdo de forma adequada, exibindo informações descontextualizadas e equivocadas, informando ao leitor que as doenças genéticas são incapacitantes, sem tratamento, associado ao retardo mental e às malformações físicas. É evidente a presença da abordagem biomédica, como por exemplo: “Os conteúdos de genética humana também poderiam ser ensinados com o objetivo de esclarecer [...]conceitos e [...] não apenas para informar. Muitas pessoas têm ideias equivocadas em relação ao risco de terem filhos com doença genética [...] que toda doença genética causa defeitos físicos ou retardo mental, são incuráveis ou intratáveis [...]" (D2, p. 36).

Os livros, na maioria das vezes, mostram aos alunos a realidade como sendo única para todos, conduzindo a formação do pensamento incorreto sobre os fatos, ao invés de apresentar uma situação que os limita, mas que pode ser transformada. Neste sentido, o conteúdo apresentado pelos livros investigados não condiz com a realidade dos alunos, ou seja, estão descontextualizados. Segundo Chassot (2000), a maioria dos conteúdos que ensinamos estão desvinculados da realidade que se pretende explicar. Muitos conteúdos de Biologia e de Ciências são mais úteis para a realização de exercícios de memorização do que para entender a vida. 
D3 propõe analisar a existência e abrangência de informações referentes à saúde bucal nos LDs de Ciências do PNLD 2004, além de apostilas adotadas pelas escolas. Foram investigados 251 livros e materiais didáticos, sendo 68 LDs de escolas públicas, em que apenas 21 apresentaram a temática em questão e, dos 183 exemplares entre livros e apostilas de escolas particulares, apenas 6 abordavam o tema.

A presente pesquisa apresenta a abordagem comportamental de saúde, trazendo uma discussão para educar, orientar e conscientizar. As temáticas apresentadas pelos LDs se relacionam entre saúde geral/saúde bucal, importância dos dentes, higiene dental, instrumentos de higiene bucal, visitas ao dentista, presença de cáries, tipos de dentes, uso do flúor, vindo ao encontro do objetivo desta revisão. "As cáries são perfurações que se formam nos dentes pela ação de bactérias, seres vivos microscópicos, que vivem na boca. Essas bactérias produzem substâncias ácidas ao aproveitar os restos de alimentos que ficam nos dentes. Devemos cuidar bem dos dentes fazendo várias escovações ao dia; ao levantar, ao deitar e após as refeições, usando creme dental. A boa escovação evita a formação de cáries. 'Mesmo que você não esteja sentindo dor, procure o dentista a cada 6 meses. Ele poderá descobrir cáries ainda no início e tratá-las, ajudando a manter a saúde de sua boca" (D3, p. 42).

Conforme Castiel (2004), a abordagem comportamental está alicerçada, principalmente, em alterar os padrões individuais de exposição ao risco, por meio das chamadas “mudanças comportamentais”. Desse ponto de vista, a promoção da saúde consiste no comportamento e estilo de vida dos sujeitos. Ele ressalta a necessidade da reavaliação, pelos autores, das informações originadas dos LDs, uma vez que a Educação em Saúde trabalha com o conhecimento no processo de mudança de comportamento dos alunos para a promoção da saúde.

D4 investiga dentro do contexto escolar, por meio de relatos de professores e diretores, ocorrências, atividades preventivas e conteúdos sobre acidentes infantis em 44 exemplares de LDs de Ciências de $1^{\mathrm{a}}$ a $8^{\mathrm{a}}$ série. $\mathrm{O}$ pesquisador ressalta que: "O tema fica restrito apenas a matéria Ciências, com ausência do tema em diversas séries e, quando presentes, estão localizados nos finais dos livros na maioria dos textos" (D4, p. 73).

Aconselha para a ampliação dos estudos sobre este tema: “[...] apesar de contemplados nos Parâmetros Curriculares Nacionais, a prevenção de acidentes e a promoção de saúde parecem ainda 
não estarem adequadamente apresentadas pelos livros didáticos" (D4, p. 73). Succi, Wickbold e Succi (2005, p. 78) discutem sobre as informações apresentadas nos LDs: "[...] perdem a oportunidade de introduzir conceitos corretos e adequados [...] na época em que as crianças estão ávidas por novos conhecimentos e podem transmiti-los para seus familiares".

D4 salienta alguns dados importantes a serem refletidos: dos 44 livros analisados, do total de 7924 páginas, apenas 39 apresentaram informações sobre acidentes ou sua prevenção. Destacando-se a constante presença da abordagem biomédica de saúde, como nestes exemplos: "[...] trabalhavam apenas o tópico de plantas venenosas ou tóxicas [...]", “[...] apenas traz um poema que fala sobre queimadura com o frio [...]. (D4, p. 68). Ainda, nestes trechos de D4, percebe-se uma tímida presença da perspectiva comportamental em um dos LDs analisados durante a investigação: "[...] trabalha algumas situações de risco (atravessar a rua, contato com animais, cuidado com cães na rua, nadar em piscinas, cuidado com fio elétrico e preparo da comida pela criança)", "[...] diversos tipos de acidentes, com plantas, com animais, como trânsito, com o uso de pipas e cuidados para prevenir" (D4, p. 68-69).

Podemos observar que nestes trechos há indicações de mudanças de comportamento no ato de promover a saúde, característico da abordagem comportamental. Considerando o livro como sendo ainda a principal referência para muitos professores e alunos é lamentável que a abordagem deste tema seja ainda tão discreta, principalmente quando se trata de um assunto tão desafiador como acidentes infantis. Desta forma, o autor ressalta a relevância de uma "abordagem mais comunitária" (HARADA; PEDROSO; VENTURA, 2005) promovendo um estilo de vida saudável.

Neste contexto, o professor, como mediador e desencadeador das mudanças de pensamento, deve trabalhar diretamente com a criança e indiretamente com os pais ou responsáveis sobre os acidentes, contribuindo para o desenvolvimento das pessoas e dos grupos pessoais (BRASIL, 2002).

Em D5, a autora busca compreender as potencialidades e limites do ensino das Doenças Sexualmente Transmissíveis (DSTs), nos LDs de Biologia nas percepções de professores e de alunos do Ensino Médio de uma escola da região metropolitana do Rio de Janeiro. Foram investigados oito LDs de Biologia, sendo estes participantes do Programa Nacional do Livro Didático Para o Ensino Médio (PNLEM) 2009. 
As DSTs, apesar de consideradas um tema relevante, ou ainda um problema de saúde pública, no contexto escolar estão restritas ao Ensino de Ciências e Biologia. Nessa perspectiva, as DSTs são trabalhadas de forma conteudista e, na grande maioria das vezes, associadas à reprodução humana nos LDs. "Quando se fez a interface entre o tema das DST e o uso de métodos de barreira física (camisinhas masculina e feminina) como prevenção e contracepção, esta ocorreu de forma muito resumida e sem maiores detalhes de prevenção de DST na sessão de métodos anticoncepcionais" (D5, p. 83).

Heilborn (2003) articula esta discussão afirmando que a inserção das DSTs em sala de aula não se refere somente a discutir as capacidades reprodutivas do ser humano, nem apenas como funcionalidade corporal derivada de reações biológicas. Durante a investigação, a autora destaca a presença da abordagem biomédica, de forma a contemplar a prevenção de doenças e não a promoção da saúde, quando discute a importância do uso da camisinha nas relações sexuais: "[...] ela é fundamental também na prevenção de muitas doenças sexualmente transmissiveis (DST), especialmente a AIDS. [...] devemos nos cuidar e nos conscientizar dos perigos dessa doença" (D5, p. 79).

Outra abordagem biomédica estava relacionada ao conteúdo sobre Seres Vivos. Além de ser simplista, foi a única relação saúde e seres vivos encontrada durante as análises dos LDs de Biologia.

"[...]micose como uma doença causadora da candidíase bucal denominado de sapinho comum em crianças e que as mesmas espécies causam candidíase vaginal” (D5, p. 84).

Rocha, Schall e Lemos (2010) discorrem sobre a abordagem biomédica como “abordagem preventiva" em meio à atuação da Educação em Saúde, sendo uma visão higienista que se caracteriza por informações básicas sobre os problemas de saúde com um alcance limitado à aprendizagem sobre doenças.

A relação entre as DSTs e mudanças de comportamentos e estilos de vidas que visam à promoção da saúde são "deficientes", segundo a autora de D5. Desta forma, ela enfatiza que informações obtidas através de outras fontes, ausentes nos LDs, podem auxiliar na compreensão dos conceitos fundamentais sobre DSTs. 
D6 realizou uma análise crítica do processo da promoção da alimentação saudável, propiciando a construção de hábitos alimentares saudáveis, relacionando ao conteúdo do currículo escolar através da análise dos LDs, assim como percepções dos professores e dos estudantes de uma turma da Educação Básica do município de Porto Lucena/RS.

O estudo estabeleceu uma investigação em 20 LDs das disciplinas de Matemática, Português, Ciências, História e Geografia, identificando que apenas os cinco LDs de Ciências disponíveis na escola continham o conteúdo de alimentação e saúde. As doenças relacionadas aos hábitos alimentares e estilo de vida inadequado estavam presentes nos quatro últimos anos do Ensino Fundamental.

De acordo com a autora, a abordagem biomédica é a mais evidenciada pelos LDs, o que não favorece a promoção da saúde, pois precisa articular mudanças de comportamentos e melhoria da qualidade de vida dos sujeitos (SILVA; FONSECA, 2009). Assim, ela define como sendo uma provocação à superação do modelo tradicional de saúde, ausência da doença e sua prevenção, passando a ponderar todos os fatores que cercam o sujeito e seu cotidiano.

"O ensino de saúde é ainda centrado basicamente na transmissão de informações sobre como as pessoas adoecem, os ciclos das doenças, os seus sintomas e as formas de profilaxia" (D6, p. 78).

Em D7, a autora analisa quais as abordagens de saúde se fazem presentes nos conteúdos relacionados à saúde da população negra em LDs de Biologia recomendados pelo PNLEM 2015.

Apenas na última década, a população negra passou a compor um grupo de atenção à saúde (BRASIL, 2009). As recentes reformas da Lei de Diretrizes e Bases da educação permitiram a inclusão das relações étnico raciais nos currículos (BRASIL, 2004). A autora enfatiza a abordagem biomédica de saúde como sendo predominante nas investigações (45\%), seguida pela comportamental (28\%) e pela socioecológica (27\%).

A abordagem biomédica se restringe a uma causa, geralmente física, que afeta o indivíduo, podendo ser identificada pelas imagens. "[...] a saúde se apresenta como oposição à doença. Assim, o foco está na doença e a condição de saúde se estabelece por ações individuais, além de ilustrar, principalmente, os agentes etiológicos, as manifestações clínicas, característicos das doenças. Este tipo de imagem pode ser visto no ciclo de vida de parasitas, frequentes nos livros didáticos" (D7, p. $84)$. 
Carmo (2014) discorre sobre apenas apresentar os sintomas das doenças quando discuti-las, o que torna limitado o ensino para a prevenção de doenças. Assim, minimizando o espaço para a reflexão sobre comportamentos, estilos de vida e promoção da saúde. A abordagem comportamental está relacionada à investigação com os comportamentos e hábitos de vida do sujeito.

"A abordagem comportamental pode ser identificada em imagens mais positivas de saúde onde são apresentados hábitos de vida saudável, mudança de alimentação, prática de exercícios, dentre outros comportamentos em busca de qualidade de vida individual" (D7, p. 84). Martins (2011) comenta os hábitos de vida negativos adotados, sedentarismo: má alimentação, estresse, abuso de drogas, sendo as principais causas do adoecimento, característicos da abordagem comportamental. Logo, apenas mudanças de comportamento não dão conta da qualidade de vida, sendo insuficientes para abranger todos os fatores sociais, culturais, políticos e ambientais característicos da abordagem socioecológica, já que ela busca incluir as causas das doenças, bem como aspectos culturais, comportamentais, políticos, em que a ênfase não se configura somente no sujeito, mas no coletivo.

"[...] abordagem socioecológica, por outro lado, pode ser identificada nas imagens onde o foco está na condição de saúde e não na de doença. A saúde se estabelece quando o indivíduo possui bemestar, levando em consideração os aspectos biológicos, psicológicos, sociais, ambientais, históricos, econômicos, políticos e espirituais" (D7, p. 84). Carmo (2014) discute esta abordagem como sendo uma alternativa adequada para discutir Educação em Saúde, visando à promoção da saúde, tendo proposições coletivas e comunitárias, voltada a políticas públicas e ações governamentais.

T1 investiga como as concepções de saúde estão sendo abordadas nos conteúdos em LDs de Ciências dos anos iniciais no Brasil. Nesta pesquisa, foram analisados os 44 livros dos professores das coleções didáticas para os anos iniciais do Ensino Fundamental ( $2^{\circ}$ ao $5^{\circ}$ ano) contemplados pelo PNLD 2010, contemplando as 11 coleções aprovadas no ano. O autor destaca certa "heterogeneidade" nas concepções de saúde, com distintas abordagens do tema. Por outro lado, a maioria das coleções aborda a saúde em seus aspectos biológicos, sobretudo em oposição à doença, destacando o indivíduo. O autor enfatiza a crítica apresentada pelos Parâmetros Curriculares Nacionais (PCN) à tendência em relacionar a saúde em uma perspectiva de "biologismo", assim apresentada no documento: 
As experiências mostram que transmitir informações a respeito do funcionamento do corpo e a descrição das doenças, bem como um elenco de hábitos de higiene, não é suficiente para que os alunos desenvolvam atitudes de vida saudável (BRASIL, 1997c, p. 61).

A investigação destaca a presença da "abordagem biopsicossocial" como referência para a saúde no conjunto dos livros analisados. Nesse sentido, o discurso relacionado à saúde alia aspectos psicológicos e sociais, além dos biológicos. As coleções que apresentam esta perspectiva mostram a preocupação em versar os temas de saúde a partir de uma forma "positiva”, diferenciando-a da doença. "[...] saúde como bem-estar, a partir da perspectiva biopsicossocial, está basicamente relacionada à discussão de um conjunto de atitudes e comportamentos considerados como saudáveis (dormir bem, prática de atividades físicas, aspectos relacionais, cuidado com o ambiente, dentre outros) [...]" (T1, p. 162).

O autor discute a abordagem comportamental de saúde como uma perspectiva de estilo de vida e escolhas pessoais, buscando mudanças de comportamentos individuais, visando à promoção da saúde: "[...] para alcançarmos o mais alto grau de saúde devemos nos responsabilizar por todas as nossas ações para evitarmos acidentes. [...], mas também prevenirmos de doenças. Ir ao dentista regularmente ou tomar vacinas obrigatórias são exemplos de métodos preventivos" (T1, p. 102).

Os PCN assinalam que a educação é considerada um dos fatores mais significativos para a promoção da saúde. A Educação em Saúde, de forma contextualizada, contribui decisivamente na formação de cidadãos capazes de atuar em favor da melhoria dos níveis de saúde pessoais e da coletividade (BRASIL, 1997).

Em T2, a autora teve por objetivo analisar como a saúde está sendo abordada nos LDs, a partir de abordagens da saúde: biomédica, comportamental e socioecológica. A última, permitindo viabilizar o tratamento da saúde numa perspectiva mais abrangente e crítica. $\mathrm{Na}$ abordagem socioecológica, a saúde deve ser entendida como um conceito positivo, multidimensional, participativo e dirigido a toda a população, considerando o meio ambiente e as condições sociais em que vive (ALVES; ARRATIA; SILVA, 1996). 
A abordagem socioecológica foi evidenciada pela investigadora, em ambos os trechos, ao tratar da presença de sardas, responsabilizando o indivíduo pelos cuidados e riscos à exposição prolongada à luz solar. "[...] tanto quem tem sardas como quem não tem, devem evitar a exposição prolongada ao sol, principalmente das 10 às 16 horas, e usar sempre filtro solar [...]”. "[...] algumas profissões exigem, de seus funcionários, jornadas de trabalho ininterruptas e com exposição ao sol e, além disso, protetores solares não são um produto de fácil acesso a todos os indivíduos" (T2, p. 104)

Ao se descuidar destas ações, excluem-se comportamentos e adoção de estilo de vida saudáveis. Desta forma, ações em benefício à saúde e à qualidade de vida tendem a aconselhar atividades ou práticas de saúde. Para a autora, a abordagem biomédica, visão reducionista de saúde, foi a predominante nas coleções $(86,9 \%)$ e apenas $13,1 \%$ das abordagens foram evidenciadas como socioecológicas.

Destacamos dois trechos em que as abordagens biomédica e comportamental se fazem presentes, respectivamente: “Doença provocada pela Giardia lamblia é adquirida pela ingestão de cistos, tanto através de verduras mal lavadas quanto de água, que receberam fezes de pessoas contaminadas. Os cistos são uma forma de resistência e suportam meses em condições adequadas, umidade e escuridão" (T2, 2016, p. 96). e "[...] pensar em desenvolver práticas de saúde, estimular comportamentos saudáveis e orientar sobre estilos de vida específicos, não é possível prevenir todos os agravos à saúde, nem todas as doenças, já que existem doenças para as quais ainda não se conhece a etiologia" (T2, p. 43).

Carvalho et al. (2010) coloca a abordagem comportamental como sendo um conjunto de atitudes e práticas voltadas para a manutenção e melhoria da saúde, com um olhar diferenciado da abordagem biomédica, por não ter como principal objetivo a prevenção ou tratamento de doenças.

Mudanças lentas vêm sendo analisadas e refletidas no atual cenário brasileiro no pensar e no educar em saúde. Dentre estas mudanças, destaca-se a tímida abordagem socioecológica, caracterizada pela presença dos elementos sociais, econômicos, culturais e ambientais. Olhar para a saúde de forma mais significativa e abrangente é difícil, mas estas atitudes vão fortalecendo propostas mais amplas, buscando a promoção da saúde, em vez de apenas prevenir doenças. 


\section{CONCLUSÃO}

A investigação em LDs de Ciências e Biologia, buscando evidencias das abordagens de saúde, bem como estão sendo discutidas na literatura permitiu compreender o caminho em que a Educação em Saúde vem percorrendo no contexto educacional. A revisão bibliográfica que realizamos levou à identificação de três principais abordagens da saúde: biomédica, comportamental e socioecológica, sendo a primeira mais tradicional e fortemente enraizada no currículo, a segunda que discute sobre mudanças de comportamentos e estilos de vidas saudáveis, e a terceira que discute as ações coletivas que visam à promoção da saúde ao invés da prevenção da mesma, levando em conta todo o contexto social.

Com esse estudo, percebemos que os LDs, muito utilizados ainda em sala de aula, contribuem de forma muito frágil com conceitos e discussões mais críticas de saúde, como podemos notar a predominância da abordagem biomédica. A Educação em saúde está fortemente ligada às disciplinas de Ciências e de Biologia, cabendo salientar que, mesmo com a presença de conceitos mais abrangentes, os LDs carregam informações simplistas de saúde. o estudo mostra, ainda, que existe a preocupação em discutir saúde desde o início da escolarização.

Diante das discussões realizadas nesta pesquisa, apontamos para uma carência dentro dos Programas de Pós-graduação sobre a temática Educação em Saúde e afirmamos a necessidade da divulgação e disseminação desta temática como um todo. Entendendo que, apesar da pequena quantidade (9), estes representam o início do processo de inserção da temática no contexto acadêmico visando abrir novos campos para discussão e reflexão sobre a Educação em Saúde no contexto escolar.

Contudo, esperamos ter contribuído para o avanço da compreensão deste tema, buscando investigar nos trabalhos já publicados, o que discutem sobre a Educação em Saúde nos LDs. Partindo desta investigação e de outras já realizadas, percebe-se a necessidade da avaliação durante a escolha do LD, para a opção daquele que supere a abordagem biomédica, de modo a abarcar diversas dimensões da saúde, já que este material é o mais utilizado na prática educativa. 


\section{REFERÊNCIAS}

ALVES, E. D; ARRATIA, A; SILVA, D. M. Perspectivas histórica e conceitual da promoção da saúde. Cogitare Enfermagem., Curitiba-PR, v.1, n.2, p.2-7. jul/dez. 1996. Disponível em:

https://revistas.ufpr.br/cogitare/article/view/8727. Acesso em: 22 nov. 2019.

BARDIN, L. Análise de Conteúdo. Lisboa, Portugal: Edições 70, 2004.

BRASIL. Diretrizes Curriculares Nacionais para a Educação das Relações Étnico-Raciais e para o Ensino de História e Cultura Afro-Brasileira e Africana. Conselho Nacional de Educação. Ministério da Educação. Brasília, 2004a. Disponível em: http://portal.inep.gov.br/informacao-da-publicacao/ asset_publisher/6JYIsGMAMkW1/document/id/488171. Acesso: 20 mar. 2019.

BRASIL. Lei 5.692, de 11 de agosto de 1971. Fixa as Diretrizes e Bases para o ensino de $1^{\circ} \quad$ e $\quad 2^{\circ} \quad$ graus, e dá outras providências. Disponível em: http://www.planalto.gov.br/ccivil_03/Leis/L5692.htm . Acesso: 27 abr. 2011.

BRASIL. Ministério da Educação e do Desporto. Secretaria de Educação Fundamental. Parâmetros curriculares nacionais: meio ambiente e saúde. Brasília: MEC, v. 9, p. 128. 1997. Disponível em: http://portal.mec.gov.br/seb/arquivos/pdf/livro091.pdf. Acesso em: 22 nov. 2019.

BRASIL. Ministério da Saúde. Secretaria de Políticas de Saúde. A promoção da saúde no contexto escolar. Revista Saúde Pública, São Paulo, v. 36, n. 4, p. 533-535, ago. 2002. Disponível em: http://www.scielo.br/pdf/rsp/v36n4/11775.pdf. Acesso em: 22 nov. 2019.

BRASIL. Portaria № 992, de 13 de maio de 2009. Política Nacional de Saúde Integral da População Negra. Ministério da Saúde. Brasília - DF, mai. 2009. Disponível em: http://bvsms.saude.gov.br/bvs/saudelegis/gm/2009/prt0992_13_05_2009.html. Acesso em: 6 abr. 2019.

CARMO, J. S. 0 tratamento dado à anemia falciforme em livros didáticos de biologia. 2014. 107f. Dissertação (mestrado) - Programa de Pós-Graduação em Ensino, Filosofia e História das Ciências, Universidade Federal da Bahia - UFBA, Salvador, 2014. Disponível em: https://ppgefhc.ufba.br/sites/ppgefhc.ufba.br/files/janete_sousa_do_carmo_-_dissertacao_ o_tratamento_dado_a_anemia_falciforme_em_livros_didaticos_de_biologia.pdf. Acesso em: 22 nov. 2019.

CARVALHO, A. A. S.; CARVALHO, G. S. Efeito da formação nas concepções de saúde de Promoção da Saúde de estudantes do ensino superior. Revista Portuguesa de Saúde Púbica, v. 28, n. 2, p. 161-170, 2010. Disponível em: http://www.scielo.mec.pt/scielo.php?script=sci_arttext\&pid=S0870-90252010000200007. Acesso em: 22 nov. 2019.

CARVALHO, G. S; DANTAS, C; RAUMA, A-L, et al. Health educacion approaches in school textbooks of 16 countries: biomedical model versus healt promotion. In: Proceedings of the ISTOE: international meeting on critical analysis of school science textbook. University of Tunis, Tunis, v.7, n. 10, p. 380-392, fev. 2007.

CASTIEL, L. D. Promoção de saúde e a sensibilidade epistemológica da categoria 'comunidade'. Revista de Saúde Pública, São Paulo, v. 38, n. 5, p. 615-622. 2004. Disponível em: http://www.scielo.br/scielo.php?script=sci_arttext\&pid=S0034-89102004000500001. Acesso em: 22 nov. 2019.

CHASSOT, A. Alfabetização científica: questões e desafios para a educação. Ijuí: UNIJUÍ, 2000. 432p.

DELIZOICOV, N. C. Ensino do Sistema Sanguíneo Humano: A dimensão histórico epistemológica. In: Estudo de História e Filosofia das Ciências- Subsídios para aplicações no Ensino. SILVA, C. C. (Org.). São Paulo: Livraria da Física, p. 265-286. 2006.

HARADA, M. J. C. S; PEDROSO, G. C.; VENTURA, R. N. A comunidade segura. Jornal da Pediatria. Rio de Janeiro, v. 81, n. 5, p. s137-s145, nov. 2005. Suplemento. Disponível em: http://www.scielo.br/pdf/jped/v81n5s0/v81n5Sa03.pdf. Acesso em: 22 nov. 2019.

HEILBORN, M. L. Articulando gênero, sexo e sexualidade: diferença na saúde. In: GOLDENBERG, P.; MARSIGLIA, R. M. G.; GOMES, M. H. A. (Orgs.). o Clássico e o novo: tendências, objetos e abordagens em ciências e saúde. Rio de Janeiro: Fiocruz, p. 197-207, 2003. 
LÜDKE, M; ANDRÉ, M. E. D. A. Pesquisa em educação: abordagens qualitativas. São Paulo: EPU, 2013.

MARTINS, L. A. C.; BRITO, A. P.O.P. M. A História da Ciência e o Ensino da Genética e Evolução no Nível Médio: um estudo de caso. In: Estudo de História e Filosofia das Ciências- Subsídios para aplicações no Ensino. SILVA, C. C. (Org.). São Paulo: Livraria da Física, 2006. p. 381.

MARTINS, L. Abordagens da saúde em livros didáticos de biologia: análise crítica e proposta de mudança. 2017. 165 f. Tese. (Doutorado em Educação) Ensino, Filosofia e História das Ciências. Universidade Federal da Bahia, Bahia. Disponível em: https://repositorio.ufba.br/ri/handle/ri/22536. Acesso em: 22 nov. 2019.

MARTINS, L. Saúde no contexto educacional: as abordagens de saúde em um livro didático de biologia do ensino médio largamente usado. 2010. 172f. Dissertação (mestrado) - Programa de Pós-Graduação em Ensino, Filosofia e História das Ciências, Universidade Federal da Bahia - UFBA, Salvador, 2011. Disponível em:

https://ppgefhc.ufba.br/pt-br/saude-no-contexto-educacional-abordagens-de-saude-em-um-livro-didatico-debiologia-do-ensino-medio. Acesso em: 22 nov. 2019.

MARTINS, L.; SANTOS, G. S.; EL-HANI, C. N. Abordagens de saúde em um livro didático de Biologia largamente utilizado no Ensino Médio Brasileiro. Investigações em Ensino de Ciências (Online), v. 17, p. 249-283, 2012. Disponível em: https://www.if.ufrgs.br/cref/ojs/index.php/ienci/article/view/215. Acesso em: 22 nov. 2019.

MOHR, A. A natureza da educação em saúde no ensino fundamental e os professores de ciências. 2002. $410 f$. Tese (doutorado) - Universidade Federal de Santa Catarina, Florianópolis, 2002. Disponível em: https://repositorio.ufsc.br/xmlui/handle/123456789/83375. Acesso em: 22 nov. 2019.

ROSA, M. D. O livro didático, o currículo e a atividade dos professores de Ciências do Ensino Fundamental. Revista Insignare Scientia - RIS, v.1, n.1, p. 1-20, 2018. Disponível em:

<https://periodicos.uffs.edu.br/index.php/RIS/article/view/7664>. Acesso em: 27 jan. 2021.

SILVA, E. C. R.; FONSECA, A. B. Abordagens pedagógicas em educação alimentar e nutricional em escolas no Brasil. Rio de Janeiro: Ed. Universidade Federal do Rio de Janeiro/Núcleo de Tecnologia Educacional para a Saúde, 2009. Disponível em: http://posgrad.fae.ufmg.br/posgrad/viienpec/pdfs/1694.pdf. Acesso em: 22 nov. 2019.

SUCCI, C. M.; WICKBOLD, D.; SUCCI, R. C. M. A vacinação no conteúdo dos livros escolares. Revista da Associação Médica Brasileira, São Paulo, v. 51, n. 2, p. 75-79, mar/abr. 2005. Disponível em:

http://www.scielo.br/scielo.php?pid=S0104 42302005000200013\&script=sci_abstract\&tlng=pt. Acesso em: 22 nov. 2019.

TESSER, C. D.; LUZ, M. T. Uma introdução às contribuições da epistemologia contemporânea para a medicina.

Ciência e Saúde Coletiva, v. 7, n. 2, p.363-372, 2002. Disponível em: http://www.scielo.br/scielo.php?pid=S1413-

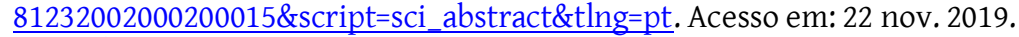

UNIJUÍ, 2000. 432p.

VASCONCELOS, S. D.; SOUTO, E. O livro didático de ciências no ensino fundamental: Proposta de critérios para análise do conteúdo zoológico. Ciência \& Educação, v. 9, n.1, p. 93-104, 2007. Disponível em: http://www.scielo.br/pdf/ciedu/v9n1/08. Acesso em: 22 nov. 2019.

WESTPHAL, M. F. Promoção da saúde e prevenção de doenças. In: CAMPOS, G.W.S; MINAYO, M.C.S; AKERMAN, M; DRUMOND JÚNIOR, M; CARVALHO, Y.M.(Org.). Tratado de saúde coletiva. São Paulo: Hucitec, Rio de Janeiro: Ed. Fiocruz, 2006. p.635-667.

ZANCUL, M. S.; COSTA, S. S. Concepções de professores de Ciências e de Biologia a respeito da temática Educação em Saúde na escola. Experiências em Ensino de Ciências, Mato Grosso, v. 7, n. 2, p. 67-75, ago. 2012. Disponível em: http://if.ufmt.br/eenci/artigos/Artigo_ID183/v7_n2_a2012.pdf. Acesso em: 22 nov. 2019.

(cc)) BY

Este trabalho está licenciado com uma Licença Creative Commons - Atribuição 4.0 Internacional. 